\title{
Super-unsupervised text classification for labeling online political hostility
}

Stig Hebbelstrup Rye Rasmussen, Alexander Bor, Mathias Osmundsen, and Michael Bang Petersen

Department of Political Science, Aarhus University, Bartholins Alle 7. Email: stighebbelstrup@ps.au.dk

\begin{abstract}
We live in a world of text. Yet the sheer magnitude of social media data coupled with a need to measure complex psychological constructs have made this important source of data difficult to use. Often, researchers engage in costly hand-coding of thousands of texts using supervised techniques or rely on unsupervised techniques where the measurement of predefined constructs is difficult. We propose a novel approach that we call super-unsupervised learning and demonstrate its usefulness by measuring the psychologically complex construct online political hate based on a large corpus of tweets. The approach accomplishes this feast by combining the best features of supervised and unsupervised learning techniques: measurements of complex psychological constructs without a single labelled data source. We first outline the approach before conducting a diverse series of tests of (i) face validity, (ii) convergent and discriminant validity, (iii) criterion validity, (iv) external validity and (v) ecological validity.
\end{abstract}

Keywords: Natural Language Processing (NLP); supervised learning; unsupervised learning; social media 


\section{Introduction}

As our lives move to the world of online social media networks, concern is mounting over the detrimental effects of online hostility for constructive democratic conversations. Yet researchers are faced with a critical challenge: How to define and measure the important phenomenon of online hostility? The problem is not trivial. As Siegel (2020, p59) concludes in her comprehensive review of the literature on online hate, the "absence of clear and consistent definitions of hate speech (...) has meant that (...) our knowledge of the causes, consequences, and effective means of combating online hate speech remains somewhat clouded by definitional ambiguity" and, furthermore, "there is no consensus with regard to the most effective way to detect it across diverse platforms." In practice, these issues have forced most researchers to identify hate speech by turning to crude "binary classification task [...] concerned with coding a document as 'hate speech or not'". Siegel (2020) focuses on "hate speech" in her review, but the issues concern all attempts to measure online hostility, not just "hate speech". Accordingly, what we propose is not a specific measure of "hate speech" but a general approach to measure online political hostility, defined here as, degrading, harassing and intimidating online content grounded in political differences.

To remedy these shortcomings, we propose that the study of online political hostility in an era of near-infinite amounts of online text on social media requires a new approach that can costeffectively measure and define online political hostility. The purpose of this manuscript is to outline and empirically validate such an approach.

Prior research on political hostility on social media has followed one of three main paths for measuring online hostility as it is expressed through text (e.g., comments and posts, tweets and retweets). The first one is based on a supervised text classification paradigm (Cranmer and Desmarais 2017). It starts by meticulously hand-labeling a large batch of texts into "hostile" and "not hostile". These hand-labeled texts then serve as the foundation of a classifier trained to "learn" and predict whether new texts are "hostile" or not. This approach thus seeks to generalize from a partially labeled corpus to the rest of a corpus. A prime example of this supervised approach can be found in Djuric et al. (2015), who use word vectors as features to classify online comments into the categories "clean" and "abusive."

The second approach is based on an unsupervised text classification paradigm, such as topic modeling, where "topics" are not known beforehand but are learned along the way (Blei, $\mathrm{Ng}$, and Jordan 2003; Grimmer 2010; Roberts et al. 2013). ${ }^{1}$ Unlike supervised classification, this approach does not require prelabeled data. Instead, it starts with the texts themselves, attempts to label them externally, and uses word co-occurrences to uncover which hateful themes and topics are present in the set of texts. For instance, Törnberg and Törnberg (2016) use this approach to study online hostile discussions focusing on Muslims in Sweden.

The third and final approach is based on dictionaries, like sentiment dictionaries (Stine 2019), or specially curated dictionaries of swear words. These dictionaries are then used to score or filter the texts based on a predefined dictionary. A good example of this approach is the study by Rodriguez, Argueta, and Chen (2019), who use sentiment and emotion dictionaries to study hate speech on Facebook.

Unquestionably, all three approaches to text classification have merits and have cross-fertilized and inspired each other (e.g. Siegel et al. (2021). However, as we explain below, each approach faces unique challenges that weaken the ability to measure online hostility. We argue that the ideal measurement of political hostility should be an approach (1) that can measure complex externally defined constructs at low cost, (2) that reflects users' own understanding of political hostility, and (3) that is able to include contextual information.

In this manuscript, we offer a novel solution to these trade-offs. Our approach makes use of so-called "word embeddings" to identify what social media users themselves find politically hostile.

1. And sometimes a combination of approaches, see e.g. Siegel et al. (2021). 
Critically, however, it goes further than typical word embedding analyses in that it uses these to construct a labelled data set for the entire corpus of text without a single hand-labelled text. Typical applications of word embeddings use the embeddings directly to e.g. track changes in the relative positions of words over time (Garg et al. 2018), or as input to a classifier (Djuric et al. 2015). We call this method a "super-unsupervised text classification" technique to signify the use of elements from current supervised and unsupervised approaches to studying online political hostility.

This manuscript consists of two major steps. First, we outline a set of general principles for the ideal measurement of political hostility and discuss how best to live up to them. Second, to validate our approach, we rely on a unique research design combining survey data with scraped behavioral data from a diverse sample of Twitter users. This distinctive research design allows us to investigate the (i) face validity, (ii) convergent and discriminant validity, (iii) criterion validity, (iv) external validity and (v) ecological validity of our approach demonstrate its advantages over current stateof-the-art methods. Importantly, these data sources are only important for validating this approach. The approach itself only requires text data and, hence, can be utilized for the entire range of text data available to political scientists studying online hostility.

\section{The ideal measurement of online political hostility}

In our view, the ideal approach to measuring online political hostility - and other complex psychological constructs - is characterized by three key features:

1. It can measure complex, externally defined constructs related to online political hostility at a low cost.

2. It can measure constructs that reflect the online users' own understanding rather than the raters' understanding.

3. It can include contextual information.

Admittedly, these features are impossible to satisfy fully but are virtues to aspire to. Nonetheless, we believe they are important to highlight since these standards are often not discussed explicitly, and, more importantly, current approaches to studying political hostility fall short compared to the method we are suggesting here. We will discuss each feature in turn and explain how current approaches attempt to deal with them. After this, we outline our preferred approach and explain how it aims to comply with these standards.

\subsection{Measuring complex externally defined constructs at a low cost?}

Researchers taking a hypothetico-deductive approach mostly care about constructs grounded in theory or conceptual work. From this perspective, the ability to classify and categorize a corpus of data is essential to the study of online hostility. In supervised text classification, this feat is often accomplished by teaching raters how to manually "label" texts according to predefined categories such as "hate speech", "offensive language" and "neither" (e.g., Davidson et al. 2017). A closely related strategy for text categorization uses dictionaries to score or filter texts (e.g., Rodriguez, Argueta, and Chen 2019; Siegel et al. 2021). Unsupervised approaches, like the one taken by Törnberg and Törnberg (2016), cannot directly classify texts based on a priori relevant constructs, such as whether a text is politically hostile or not. Using topic modeling to classify tweets into "politically hostile" and "not politically hostile," researchers would have to rely on the topic that comes closest to this external labeling; most likely, no single topic, or perhaps no topic at all, would directly fit this externally defined categorization.

One of the main problems with current approaches to measuring externally defined constructs is cost. Hiring student assistants or persuading other researchers to hand-label text is financially costly 
and exceedingly time consuming. It takes time and effort to design the coding scheme, repeatedly refine it to meet issues that will likely arise, and, of course, to actually read and code large chunks of text. These costs, furthermore, limit the complexity of the constructs that are typically measured. In principle, a coding scheme can be used to classify documents along multiple dimensions. But the tedious work involved in hand-coding text means that the typical application has to fall back on a single dimension such as "hostile" or "not hostile." This problem is pertinent when using data from social media where texts often come in millions and sometimes billions. Hand-labeling even a small percentage of these is a massive undertaking.

\subsection{Using measures that reflect online users' own understanding rather than the raters' under- standing?}

Measures of psychological constructs should be ecologically valid. In our case, this implies that texts coded as "hateful" are perceived as such by social media users themselves. Constructing ecologically valid measures is difficult without grounding the classification process in users' own experiences. This problem is especially pertinent to studying a phenomenon such as online hostility. Beliefs about what hostile speech is likely fluctuate across individuals and national contexts (Siegel 2020). Failing to start from the users' own understanding of the construct of political hostility also risks relying on the (subjective) and potentially biased labels of external raters. For instance, Wich, Bauer, and Groh (2020) recently demonstrated that the political bias of raters systematically impacted the classification of hate speech. Relatedly, the high costs associated with hand-labeling (see Section 2.1) means that the number of raters per text is typically low, ranging from one to four (Barberá et al. 2021; Davidson et al. 2017). While internal reliability can be investigated by reporting interrater reliability coefficients, this approach still rests on the work of only a few people's informed but subjective evaluations of whether, say, a given tweet is politically hostile. As researchers and their raters may reflect a narrow subset of the population in terms of racial, sociodemographic or political diversity (e.g., Duarte et al. 2015), this may bias ratings, even when ratings are internally consistent. When studies present classifications of hate and hate speech based on the work of a few raters without disclaiming the ideological background of these same raters, we are left uncertain about the amount of bias present in the labeling exercise.

The topic modeling approach used by Törnberg and Törnberg (2016) is based on the logic that if two words co-occur often in a topic, it is because respondents themselves perceive that these words tend to go together. The topics thus do not necessarily reflect the researcher's perception of the content of a construct but rather the respondents' subjective perceptions. Using external coders, such as in the study by Davidson et al. (2017) where the authors label a dataset, or using an externally defined dictionary provides no such reassurance, which is a good reason to prefer the approach taken by Törnberg and Törnberg (2016).

\subsection{Can contextual information be included when studying online hostility?}

The final ideal to aspire to when measuring online political hostility concerns context. Researchers studying online hostility are often interested in comparing their constructs across political groupings - for example, do Democrats and Republicans share common beliefs about what is hostile? - or even across countries. Is the construct of political hostility even the same across political groupings? Preliminary studies by Wich, Bauer, and Groh (2020) suggest it is not, since raters are biased in labeling across the political spectrum. Furthermore, researchers of online hostility could be interested in comparing macro-level factors of online hostility such as inequality or political polarization, which is very difficult to do using dictionaries or hand-labeling.

Incorporating contextual information in the topic modeling approach used by Törnberg and Törnberg (2016) is fully possible. In structural topic modeling, for example, the topic prevalence or topic content (Roberts et al. 2013) can be modeled to examine whether certain topics are more 
prevalent among particular groups, such as Democrats compared to Republicans. Conversely, it is difficult to incorporate contextual information in the supervised classification approach of, for example, Davidson et al. (2017) and Djuric et al. (2015). If implicit biases (Wich, Bauer, and Groh 2020) make it difficult to classify online hostility within a country, studying online hostility across countries would seem a herculean task. And how do we account for measurement invariance, that is, making sure that the constructs we are comparing across contexts are even the same? If we were relying on a latent construct based on survey data, we could of course test for measurement invariance (Meredith 1993). To our knowledge, however, such tests or procedures have not been developed for hand-labeling of constructs across contexts for text data.

\subsection{Super-unsupervised text classification}

Current approaches to studying online hostility each have distinct advantages and disadvantages. Our contribution is to demonstrate that the use of so-called "word embeddings" and the choice of focal words offer a simple and cost-effective, yet elegant solution to the problems of defining and measuring complex constructs like political hostility. Since the method draws on both supervised and unsupervised techniques, we label it a "super-unsupervised" approach. Consistent with an increasing number of political science studies (Rheault and Cochrane 2020; Rodman 2020), we use word embeddings to identify what social media users themselves find politically hostile, but, critically, we go further than typical word embedding analyses by using these to label the entire corpus of text.

Specifically, the "super-unsupervised" technique involves six simple steps: (i) preprocess the text data; (ii) train word vectors for the desired corpus of text (e.g., a collection of tweets and retweets); (iii) choose a construct (e.g., "political hostility"); (iv) average the word vectors contained in each document in the corpus (e.g., a tweet or retweet) and calculate the distance to the focal construct; (v) sort by the calculated distance; and (vi) appreciate the final "labeled" dataset. ${ }^{2}$ This six-step procedure is illustrated in Figure 1. In a typical classification setting where the word vectors are used as features for a classifier (e.g. Djuric et al. (2015)), only the first two steps are used.

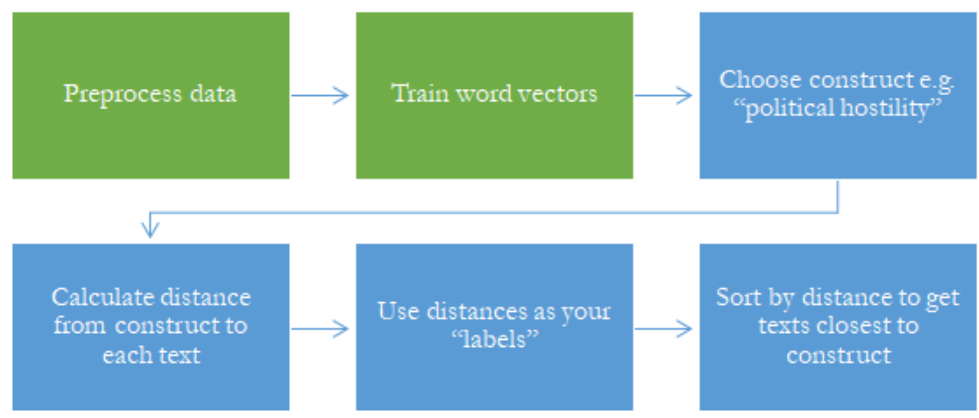

Figure 1. Illustration of the super-unsupervised approach. The colors refer to how the proposed approach differs from traditional approaches using word embeddings. The "green" boxes are very typical when using word embeddings, whereas the blue boxes reflect the novel contribution of the super-unsupervised approach

2. More specifically we use the word "political" and "hate". We prefer the word "hate" over "hostility" since this word is used more often in everyday language, which our method relies on, compared to the more technical term "hostility". A second reason for choosing to call our measure for "online political hostility" rather than "online political hate" is to not conflate it with the more specific term "hate speech", which we are not purporting to measure. As discussed below, and illustrated in figure 1 in appendix 3 the method is quite robust to the specific choice of words. 
Researchers have started using word embeddings in important studies on, for example, predicting the ideological placements of political parties (Rheault and Cochrane 2020) and investigating the changing meaning of political concepts over time (Rodman 2020). Accordingly, we are certainly not the first to use word embeddings to study political texts, nor are we claiming to be the first to use word embeddings to classify hostile tweets (e.g., Djuric et al. 2015).

The present contribution is, however, that by following the approach outlined in Figure 1, word embeddings can be an exceptionally powerful tool for labeling even extremely large datasets. To our knowledge, no one has used word embeddings to accomplish this goal before. Moreover, we suggest that using this technique will bring us closer to satisfying the three standards outlined previously. Table 1 summarizes the advantages of the super-unsupervised approach compared to traditional approaches to measuring online hostility. We will briefly elaborate on each element in turn.

Table 1. Overview of similarities and differences between dictionary based, topic modeling based and the superunsupervised approach towards measuring and defining political hostility

\begin{tabular}{c|c|c|c|c}
\hline Standard & Dictionary based approach & Topic modeling approach & Classification approach & Super unsupervised approach \\
\hline $\begin{array}{c}\text { Able to measure complex } \\
\text { externally defined constructs? }\end{array}$ & Yes & No & Yes & Yes \\
$\begin{array}{c}\text { Constructs reflect user's self-understanding and } \\
\text { not the understanding of raters }\end{array}$ & No & Yes & No & Yes \\
\hline \begin{tabular}{c} 
Can include contextual information? \\
\hline
\end{tabular} & No & No & No & Yes \\
\hline
\end{tabular}

\subsubsection{Super-unsupervised learning and the three standards for measurement and definition of on- line hostility}

The super-unsupervised approach utilizes the so-called word2vec methodology, and an in-depth understanding of the approach requires an understanding of both this methodology and of language itself. Briefly, the word2vec methodology rests on the so-called "distributional hypothesis," explained by Firth (1957): "You shall know a word by the company it keeps." The organizing idea is that the meaning of words is not entirely defined by some logical structure of the language itself (langue) but rather in its actual usage and everyday understanding (parole) (De Saussure, 2011[1916]).

The idea of using natural language and written words to conduct serious measurement is not as novel as it first might appear. In fact, this method propelled the widespread success of the five-factor model of personality (i.e., the Big Five personality traits). The key idea is that if a personality trait is salient - that is, an important and enduring feature in a society - it will be captured by specific words (Matthews, Deary, and Whiteman 2003, chapter 1). This means that by consulting a lexicon, we can arrive at a precise picture of which personality traits are important in a society; an approach termed the "lexical hypothesis." Next, researchers took large quantities of verbal descriptors of real humans and applied factor-analytic methods to analyze which trait words correlated. This was done by simply asking people whether specific words, like "enthusiastic" or "assertive", were something that described them. It emerged that words like "enthusiastic" and "assertive" tend to go hand-in hand; a person described as "enthusiastic" also tends to be described as "assertive." Super-unsupervised learning builds on the same insight. If a construct is salient, it is likely that dedicated words have been invented to describe it by the users of a language.

A word thus derives meaning from its context and, hence, the other words surrounding it. Words that tend to co-occur are more often than not similar in meaning. In essence, the approach of word embeddings works by projecting each word onto a multidimensional space, providing a word vector for each word in a corpus. The distance between words are then based on how often they tend to co-occur. If the words co-occur often, their distance is small; if the words co-occur infrequently, their distance is large. In this way, the approach can calculate the distance between different words like "Trump" and "hate," or the distance between a tweet (by averaging the words 
within it) and a construct such as "hate". Importantly, these distances are based on the document authors' (e.g., the person who is tweeting) own perceptions of what is related to, for example, hate and hostility. Furthermore, since this technique does not require labels, it is completely unsupervised. Accordingly, it has the same (often useful) properties that topic modeling has: Labeling is not done by only a few expert raters, and the constructs are defined by respondents.

Collectively, these features make word embeddings immensely useful for measuring political hostility in a manner that complies with the three standards presented above. Word embeddings (1) automatically generate labels to measure complex, externally defined constructs at a low cost that (2) reflect the users' own understanding. To derive all political texts in a corpus, a researcher would simply use the word vector for the word "political" and calculate the distance from this vector to a text. The closer the text is to the "political" vector, the greater the likelihood that the text is a political text. Further, we can also straightforwardly use word vectors to (3) incorporate contextual information, our third standard for measurement of online hostility. A simple way to do this is to train word embeddings separately for the contexts (e.g., for Democrats and Republicans separately) before aligning the vector spaces. We elaborate on this in appendix 1.

These three standards concern the measurement of political hostility. But the super-unsupervised approach also has a huge advantage when it comes to the definition and conceptualization of political hostility. Scholars disagree fiercely over how exactly to define political hostility: Is it the use of offensive and aggressive language in online discussions, "hate speech" aimed at marginalized and vulnerable groups (Waldron 2012) or cyber-bullying (Griezel et al. 2012)? Surely the categories used by Davidson et al. (2017) - that is, "Hate speech", "Offensive language", and "Neither" - and the categories "Clean" and "Abusive" used by Djuric et al. (2015) measure quite distinct constructs. Our solution to this very difficult but important problem is simple: Rely on the users' own perceptions of what constitutes online hostility. If the users find that a text is hateful (i.e. has a low distance to our hate vector), we simply take their verdict at face value.

The simple nature of our approach may concern some. A skeptical reader may interject: "Are you actually measuring politically hostile rhetoric, or are you simply measuring whether a social media user is blaming others of being hostile?" An example of the latter could be a tweet saying "Democrats are so hateful", which is obviously not measuring hatred, whereas the tweet "I hate all Democrats" would be an example of the former. However, this would be a misunderstanding of how the word vectors are created. If a tweet says "Democrats are hateful", then the algorithm connects "hate" and "Democrats" since it is continuously trying to predict a word given its context words (cf. 1.1 in appendix 1). Another couple of tweets say "Neo-Nazis are racist, homophobic and filled with hate", which connects "hate" to "homophobic" and "racists". If there are more examples of the former types of tweets, "talking about hate", rather than the latter, "being hateful", the hate vector would not in general be associated with words that are (almost by definition) hateful, such as "racist" and "homophobia". ${ }^{3}$ The tweet "Democrats are racist and hateful" would be a mix of being hateful, i.e. calling another group "racists", and talking about hate at the same time. ${ }^{4}$ If there are more tweets that clearly connect "hate" to actual hate, or simply a mix of actual hate and talk about hate, compared to tweets that are only talking about hate, the algorithm would (correctly) associate "hate" with actual hate and not (primarily) talking about hate. Of course, we also subject the method to validity tests below to investigate whether the method is in fact associated with actual hate, but it is important to stress that the method is, by itself, likely to measure actual hate rather than talk about hate based on the simple "distributional hypothesis": "You shall know the word hate from the company it keeps". 5

3. We show below that the word vector "hate" is in fact highly associated with the words "racist" and "homophobia".

4. Example tweet \#1 in table 2 has exactly such a sentence construction.

5. For further discussion of this point see appendix 2 


\section{Validating the super-unsupervised approach for studying online political hostility}

Our goal is to demonstrate the usefulness of the super-unsupervised approach for measuring online hostility. A crucial first step in this regard is to validate it. To this end, we use a large corpus of text data from Twitter (tweets) to benchmark our approach against multiple standard validation criteria; an approach named construct validation (Cronbach and Meehl 1955). We show that the super-unsupervised approach can be used to measure and distinguish between (1) political tweets, (2) hateful tweets and (3) politically hateful tweets (i.e. the combination of the two). To our knowledge, this is the first study that clearly demonstrates how to measure (3).

We examine face validity by extracting a number of concrete tweets classified as more and less politically hateful by the super-unsupervised approach to examine if it appears to measure what we claim it does. Next, we examine the convergent and discriminant validity by correlating the classifications produced by the super-unsupervised approach with a number of theoretically derived individual-level predictors of political hate. We examine criterion validity by extracting political actors in tweets. Then, we examine the external validity of the classifier by applying it to another dataset of Twitter posts and by directly comparing the outputs of the classifier to hand-coded labels of offensive posts on Twitter. Finally, we examine the ecological validity of the classifications by examining whether Republicans and Democrats perceive political hate differently. In particular, on the basis of psychological research, there are reasons to believe that the objects of political animosity are different for Democrats and Republicans (Brandt et al. 2014). If the measured constructs produced by the super-unsupervised approach work as intended, such differences should be evident. As discussed above, a key feature of the super-unsupervised approach is that it allows for the integration of contextual information. The full exposition of the last four validation exercises can be found in appendix 1.

\section{Data and Methodology}

To examine the construct validity of the super-unsupervised approach, we rely on a unique dataset of American Twitter users. These users were recruited by YouGov from their web panel and invited to participate in a study. The participants completed several surveys containing questions measuring their political attitudes (e.g., partisanship, political interest and knowledge), self-reported online hostility, and a range of demographic variables that we analyze below. Furthermore, the respondents provided informed consent to let us scrape their Twitter data and, hence, provided us with a unique opportunity to link survey data with social media data. The Twitter scraping began on March 4, 2019 and ran until March 30, 2020, leaving us with 5,076,851 tweets.

We use the tweets to create two word vectors: a "political" word vector and a "hate" word vector. Second, we also use the ability to calculate a combined "political hate" word vector by adding our two individual word vectors. Third, for each social media post in our corpus, we average the word vectors in each text and calculate the distance from each individual text to our three chosen word vectors i.e. "political", "hate" and "political hate". These distance measures comprise our primary measures of political hostility. If a given post has a close proximity to the "hate" word vector, we thus classify it as more hateful on average. The typical distance measure is the cosine similarity measure, see also the Supplementary Materials (SM) Section 1.1, which ranges from -1 (complete opposites) to 1 (complete similarity), with a cosine similarity of zero indicating no relationship between two vectors.

A more detailed discussion of word embeddings and our implementation can be found in SM Section 1.1. Here, we show that the super-unsupervised approach is very robust to different preprocessing choices. In addition, most of the results sections have additional information on methods and measures in SM 1 using the same headings and structure as in the main text. 


\subsection{Results}

This section discusses the results for our investigation of the construct validity of the proposed superunsupervised approach. We proceed in three steps. First, we provide simple illustrations of the face validity of the approach. Second, we provide formal tests of the convergent and discriminant validity of the outputs of the approach. Third, we provide a number of tests of the criterion validity of the approach. The last validity tests can be found in SM 1.

\section{Face validity}

\subsection{Methods and measures}

We first compare the results from our "political" word vector and our "hate" word vector to Google's Perspective API (JIGSAW). The Perspective API is a machine learning classifier trained on Wikipedia editorial discussions, and is a commonly applied method for detecting online toxicity (e.g. (Kim et al. 2021)). Accordingly, by comparing results using our supervised-unsupervised approach to the "toxicity scores" obtained from applying the Perspective API on the same set of tweets provides a straightforward way of examining whether we measure what we claim to be measuring.

\subsection{Results}

Table 2 provides a set of example tweets with the highest scores on either toxicity or political hate (the remaining tweets are displayed in Table 3 in SM Section 1.2.2). The face validity test simply concerns whether the tweets themselves are "face valid." We randomly sampled 10 example tweets from the 40,000 highest-scoring tweets for each measure. We then plotted them in a twodimensional space according to their score on the "political" word vector and the "hate" word vector in Figure 2. The third dimension, indicated by color in the figure, shows the tweets' toxicity score. The scales run from zero to one, where a higher score indicates a greater correspondence with the given dimension. Distributions for all variables can be seen in SM Section 3.

As we can see, the tweets with high toxicity scores (which are purple) are mostly about swear words, like "shit," "dick" and "fucking"; see Tweets 11, 15 and 19. There are some examples of overlap. Tweet 13, which is both relatively high on the "political" dimension and the "hate" dimension also scores high on toxicity. This makes sense since the content is both political - it is about "neolibs" and "GOP" - and is also strongly worded. Conversely, Tweet 4 has a low toxicity score but is relatively high on the "political" dimension and the "hate" dimension. This also makes sense. The tweet does not use swear words but is clearly political, i.e. about the US election, and uses aggressive wording to describe it, saying Trump committed an "attack" on the election.

In terms of distinguishing between "political" and "hate," we see that Tweets 7 and 4 are relatively close to the "political" word vector whereas they only score around .5 on the "hate" word vector. This also corresponds to their content: They are mostly political and not extremely hatefilled. Tweet 7, for example, talks about "political rivals" and "Trump" and only uses the somewhat negatively valenced word "smear." Conversely, Tweets 1 and 6 are relatively close to the "hate" word vector but not as close to the "political" word vector as Tweets 7 and 4. Again this also corresponds to their content: Tweet 1 is mostly cursing ("hate-filled, misogynoir crime") while Tweet 6 is also mostly an expression of discontent; the only part that hints at political content is the word "Trump."

We also note that although Tweet 1 contains the word "hate," Tweet 6 does not, and yet both score relatively highly on the "hate" dimension. Similarly, Tweet 7 and Tweet 4 both score relatively highly on the "political" dimension, but only Tweet 7 contains the word "political." We are thus clearly picking up on something substantively interesting that goes beyond a simple algorithm that contains a dictionary lookup of e.g. the words "hate" and/or "political." The distributions for toxicity and political hate can be found in SM Figure 1.

Table 3 shows the words most closely associated with our words vectors "political", "hate" and "political hate." It further validates the super-unsupervised approach and shows why it works better 


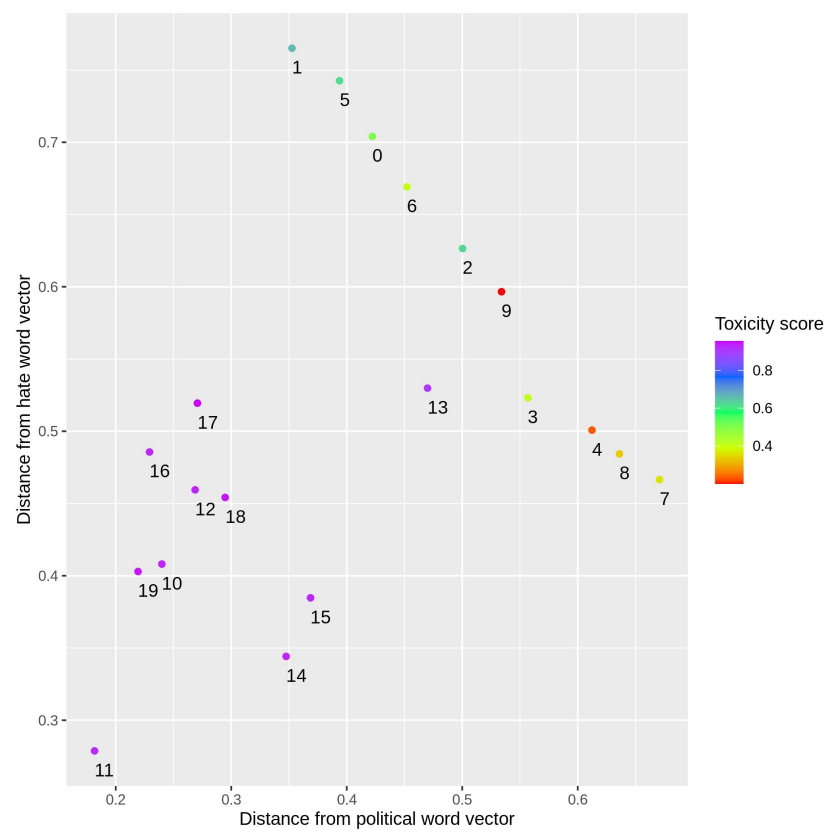

Figure 2. Sample locations for tweets in terms of their distances from the "political" and "hate" word vectors and their Toxicity score.The sample texts are 10 randomly selected tweets from among the first 40,000 tweets scoring closest to "political hate" and 10 randomly selected tweets from among the 40,000 scoring most highly on toxicity. The tweets are sorted separately for toxicity and political hate. The rest of the example tweets can be found in the SM Section 1.2.2.

Table 2. Full text for example tweets in Figure 2

\begin{tabular}{ll}
\hline Number & Tweet \\
\hline 1 & $\begin{array}{l}\text { Do not give your eyeballs to this racist, hate-filled, misogynoir crime. I Stand- } \\
\text { WithLeslie }\end{array}$ \\
\hline 4 & $\begin{array}{l}\text { I've been trying to make this fundamental point since people began talking } \\
\text { abt Trump's crime of "soliciting a foreign attack on our election." It wasn't a } \\
\text { foreign attack, it was *his* attack. https://t.co/v3Bkr5NKuK }\end{array}$ \\
\hline 6 & $\begin{array}{l}\text { Most presidents inspire people to do good things, but not Donald Trump. } \\
\text { He inspires people to do evil things and commit violent acts of terror. The } \\
\text { TrumpVideo is on brand for him. }\end{array}$ \\
\hline 7 & $\begin{array}{l}\text { @jsolomonReports Uh-oh! Now Latvia is helping Trump smear his political } \\
\text { rivals. }\end{array}$ \\
\hline 11 & $\begin{array}{l}\text { Maybe people who instantly blame Islam call for authoritarian crackdowns - } \\
\text { with no facts- are exploiting attacks for pre-existing agendas? }\end{array}$ \\
\hline 13 & $\begin{array}{l}\text { No shit Cisco and Frost are useless TheFlash } \\
\text { what "expertise" you claim has done nothing for future generations. We see } \\
\text { you. The neolibs need to go just as badly as the racist GOP. } \\
\text { 'mon kids, it's time for REAL change! https://t.co/7D2IUfm5rm }\end{array}$ \\
\hline 15 & $\begin{array}{l}\text { @crooksandliars Ridiculous for wanting a fair and honest election? sarahsil- } \\
\text { verman sucks Jamie Diamond's dick. }\end{array}$ \\
\hline 19 & $\begin{array}{l}\text { NextYearlPromiseTo continue using my blinker while driving even if everyone } \\
\text { else on the road fucking doesn't... }\end{array}$ \\
\hline
\end{tabular}

than a simple dictionary-based approach. All of the words closest to the word vectors are words we normally associate with these words. "Hate" is closely related to, for example, "hateful," "racist" and 
Table 3. Cosine similarity distances for the word vectors "political," "hate" and "political hate." For each word vector the 20 words closest to the given word are extracted

\begin{tabular}{|c|c|c|}
\hline Political & Hate & Political hate \\
\hline (1.0, political) & $(1.0$, hate $)$ & (0.83, political) \\
\hline (0.68, correctness) & $(0.78$, hatred $)$ & $(0.79$, hate $)$ \\
\hline (0.68, opponent) & (0.73, hateful) & (0.71, correctness) \\
\hline (0.68, politic) & (0.66, incite) & $(0.7$, hatred $)$ \\
\hline (0.66, rival) & (0.66, haters) & (0.69, espouse) \\
\hline (0.64, partisan) & (0.66, bigotry) & (0.69, politic) \\
\hline (0.63, apolitical) & (0.64, intolerance) & (0.69, divisiveness) \\
\hline (0.63, ideological) & (0.63, supremacists) & (0.66, hateful) \\
\hline (0.62, politically) & $(0.63$, hater $)$ & (0.65, incite) \\
\hline (0.6, influence) & (0.62, supremacist) & (0.65, ideology) \\
\hline (0.59, weaponize) & (0.62, homophobia) & (0.65, divisive) \\
\hline (0.58, cynical) & (0.62, racists) & (0.63, weaponize) \\
\hline (0.58, solely) & (0.62, foment) & (0.63, discourse) \\
\hline (0.58, partisanship) & (0.61, stoke) & (0.63, ideological) \\
\hline (0.57, weaponizing) & (0.61, racist) & (0.63, animosity) \\
\hline (0.56, weaponized) & (0.61, incitement) & (0.62, overt) \\
\hline (0.56, cartoons) & (0.61, espouse) & (0.62, intolerance) \\
\hline (0.56, legitimate) & $(0.61$, spew $)$ & (0.62, rhetoric) \\
\hline (0.56, deference) & (0.6, racism) & (0.62, promote) \\
\hline (0.55, discourse) & (0.6, supremacy) & (0.61, foment) \\
\hline
\end{tabular}

"intolerance," and "political" is closely related to "influence," "opponent" and "partisan." In a sense we are therefore not only using the words "hate" and "political"; we also use all the words close to them. This explains why we obtain substantively meaningful example tweets in Table 2 above, even when the words "hate" or "political" do not consistently appear. The approach is thus relatively insensitive to the specific words chosen; we could just as easily haven chosen the words "politics" or "hostile" and we would have obtained results that are very similar to those obtained in the analyses of correlations. "Political hate" and "Political hostility" has a cosine similarity of .75.

In fact, the results are even more robust than this since we also use subwords. Thus for each word we also include a series of subwords that include different spellings and potentially shorter forms of the same word. This also applies for each of the words in the robustness check, where we move further and further away from the chosen words. Taken together, all of these results suggest that our supervised-unsupervised approach is face valid.

\section{Convergent and Discriminant Validity}

Convergent and discriminant validity concerns whether our political hate vector correlates with what we expect it to correlate with (convergent validity) and does not correlate with anything it shouldn not (discriminant validity) (Campbell and Fiske 1959). This analysis progresses in two parts: First, we construct a series of marginal correlation tables. We expect the "political hate" vector to positively correlate with several subjective, self-reported measures from our survey; in particular, political interest and political knowledge, since politically interested and knowledgeable individuals are more likely to tweet about politics (convergent validity). In addition, the "political hate" vector should correlate positively with self-reported hostility and traditional measures of online hostility, such as sentiment scores, toxicity scores, as well as more recent types of language models (see below). At the same time, these traditional measures should be less related to the survey-based measures of political interest and political knowledge compared to our "political hate" vector (i.e. discriminant 
validity), as these traditional measures are not oriented towards politics specifically. We also include demographic information on gender since this is often discussed in terms of online hostility (Siegel 2020).

In order to compare the super-unsupervised approach with a traditional classification approach we have hand labelled 2500 tweets; this also further corroborates the convergent validity of the super-unsupervised approach. This allow us to compare the results to the state-of-the-art approach towards measuring online hate, the "binary classification task" (Siegel 2020, p59). We use hand labeling as a first step and ads a classifier as a second step.

In the second part of the analysis, we calculate a series of partial correlations to investigate the two separate dimensions of political hate, namely "political" and "hate." After partialing out the shared variance between the "political" vector and the "hate" vector the correlation between the "political" vector and hostility and toxicity should be much smaller than the marginal correlation above. Conversely, the "hate" vector should correlate much less with political interest and political knowledge when the shared variance with the "political" vector is accounted for.

\subsection{Methods and measures}

To measure sentiment, we apply the popular AFINN sentiment dictionary on our tweets (Nielsen 2011). AFINN scores rest on a dictionary approach, giving valence scores from -5 (very negative) to +5 (very positive) to each word in the dictionary. This is by no means the only way to measure sentiment, but it serves as a good benchmark to contrast our estimates with (Stine 2019). We also use Google's Perspective API toxicity scores, as mentioned above, as a second external measure of online hate.

We used four expert raters ${ }^{6}$ to rate 2500 randomly sampled tweets from the full corpus. Each rater received 500 common and 500 unique tweets and was asked to rate how hostile (five-point scale, 1 = "Not at all hostile", 5 = "Very hostile") and political (binary scale, $0=$ "Not political", 1 = "Political") each tweet was. We aggregated the ratings to create a measure of "political hate", labelled "Political hate expert" in Table 4. We use this measure to create a classical predictive model using the labelled data as input, which we subsequently used to score the rest of the tweets (see SM Section 1.3 for further details on the expert raters and the classical classification exercise). If we treat the political hate measure and the expert based measure of political hate as binary by splitting them at the mean, we get an accuracy of 74 against a baseline of 50 since our groups are balanced, which is quite good. They are thus measuring the same construct to a very large extent, and the idea that the super-unsupervised approach is merely measuring "talking about hate" is thus ill founded.

Finally, we include a measure of political hostility based on so-called pre-trained language models, which follow a similar approach of calculating the distance from "political hate" to each tweet to gauge whether the tweet is politically hostile or not. These newer pre-trained language models can take into account more advanced grammatical relationships. Whereas each word has one single location in a vector space in the super-unsupervised approach, a word's location in these models depends on the surrounding words. A prime example is the word "bank", which depending on the surrounding words, can refer either to a "river bank" or a place to withdraw and deposit money. Because of this ability, the embeddings here are sometimes referred to "contextual" embeddings (Hewitt and Manning 2019; Clark et al. 2019). We therefore term this measure "Political hate language model" in Table 4. The most famous of these is probably Google's BERT model (Devlin et al. 2018). ${ }^{7}$. The main drawback of this approach, is the amount of data needed is potentially huge, which makes it difficult to use to study changes in embeddings across, for example, Republicans and Democrats or over time as further discussed below in Section 7.

6. The raters were a mix of postdocs and $\mathrm{PhD}$ students working in the field of online hostility from INSTITUTION BLINDED but not part of this particular research paper.

7. We use a specific version of this architecture that is ideally suited to calculating (contextual) embeddings for sentences which we are interested in here. See SM Section 1.3 


\subsection{Marginal correlations with self-reported measures and expert raters}

Table 4 presents the results. Here, the first rows for each construct are the results for the annotation sample (grey), while the second row (black) are the results for the full sample. It is clear that the correlations for the tweets from the annotation exercise are slightly smaller than those in the full sample; likely because the variation is slightly smaller.

Turning to the data from the the full sample, we see that the super-unsupervised performs just as well as the "political hate classifier" and the more advanced "political hate context" classifier. The correlations between our survey based measures and these three different operationalizations of political hate are almost identical. And the correlations between these three operationalizations of political hostility is also very high, around .8 , suggesting they measure a very similar construct. Results for our "political" word vector are similarly strong: The correlation between the "political" vector and self-reported political interest and political knowledge are .45 and .48 . Even in a survey setting using only self-reported measures, a correlation of .48 is high. The correlations presented here are correlations for completely different ways of measuring political interest, i.e. self-reported political interest as measured by a survey and interest in politics measured by how often somebody engages in political discussions on Twitter. Clearly these are corroborating the strong convergent validity of the super-unsupervised approach.

The "hate" word vector also correlates positively with the expected measures: The correlation with self-reported hostility is .30, and the correlation between political hate and gender is -.13. A correlation for a binary variable such as gender is perhaps not the most intuitive way of representing the results, however. If we split the "political hate" column into six equally large segments, the proportion of females is .64 for those who are least hateful and .42 for those who are most hateful. This corresponds to previous studies demonstrating that those who produce hate speech are more likely to be male (Costello and Hawdon 2018)

The results for the "hate" word vector are fairly similar to those obtained with both the toxicity and sentiment scores; both correlate at roughly the same levels with hostility. What neither the toxicity nor sentiment scores correlate as highly with are political knowledge and political interest. If we were to use these to capture political hate, we would probably be misled since the correlations for our "political hate" word vector and political interest and political knowledge are roughly double those of the correlations that toxicity and sentiment have with these measures. Thus although the toxicity and sentiment scores seem to capture the "hate" component, they fail to capture the important "political" part of online political hate. Accordingly, these results show that our supervised-unsupervised approach has a strong divergent validity. 2 in appendix 3 suggest a sample size of 100,000 tweets is needed for our approach to work.

\section{Ecological validity: Context matters}

To illustrate the usefulness of the super-unsupervised approach, and to indirectly validate it further, we investigate its ability to take context into account. This is a test of ecological validity, that is, whether investigations are able to capture perceptions of participants in their "real-life" environments (Schmuckler 2001).

At least two avenues for research opens up using this approach. First, we can examine expressions of political hate across different contexts at a single point in time. For example, we can examine whether Democrats and Republicans express political hate differently, and whether political hate differs across countries. We know that Democrats and Republicans perceive the world differently at a fundamental level (Jost, Federico, and Napier 2009), so we could expect differences in their perceptions of political hate. Further, psychologists have recently used word embeddings to investigate the presence of gender discrimination across 45 different languages, (DeFranza, Mishra, and Mishra 2020) and it would also make sense to expect differences in political hate across countries. Second, we can study changes in political hate in the same context over time. An example of such an approach 
Table 4. Correlation table for the correlations between measures based on tweets (political hate, political, hate, toxicity and sentiment) and the measures from the survey data (political interest, political knowledge, self-reported hostility and gender. The first row for each construct, which has a gray color, are the results for the annotation sample, and the second, which has a black color, are the results for the full sample

\begin{tabular}{|c|c|c|c|c|c|c|c|c|}
\hline & $\begin{array}{l}\text { Toxicity } \\
\text { score }\end{array}$ & $\begin{array}{l}\text { Afinn } \\
\text { score }\end{array}$ & $\begin{array}{l}\text { Political } \\
\text { vector }\end{array}$ & $\begin{array}{l}\text { Hate } \\
\text { vector }\end{array}$ & $\begin{array}{l}\text { Political } \\
\text { interest }\end{array}$ & $\begin{array}{c}\text { Political } \\
\text { knowledge }\end{array}$ & Hostility & Female \\
\hline Political hate expert & 0.35 & -0.28 & 0.67 & 0.45 & 0.43 & 0.37 & 0.28 & -0.11 \\
\hline \multirow{2}{*}{ Political hate } & 0.47 & -0.32 & 0.91 & 0.87 & 0.33 & 0.35 & 0.24 & -0.07 \\
\hline & 0.67 & -0.61 & 0.94 & 0.9 & 0.41 & 0.44 & 0.3 & -0.13 \\
\hline \multirow{2}{*}{ Political hate classifier } & 0.34 & -0.25 & 0.66 & 0.43 & 0.42 & 0.36 & 0.27 & -0.12 \\
\hline & 0.57 & -0.55 & 0.81 & 0.69 & 0.42 & 0.42 & 0.28 & -0.1 \\
\hline \multirow{2}{*}{ Political hate - language model } & 0.39 & -0.3 & 0.63 & 0.47 & 0.32 & 0.33 & 0.24 & -0.13 \\
\hline & 0.59 & -0.62 & 0.84 & 0.65 & 0.43 & 0.44 & 0.27 & -0.17 \\
\hline \multirow{2}{*}{ Toxicity score } & 1.0 & -0.24 & 0.4 & 0.45 & 0.14 & 0.17 & 0.15 & 0.0 \\
\hline & 1.0 & -0.68 & 0.58 & 0.67 & 0.24 & 0.22 & 0.29 & -0.05 \\
\hline \multirow{2}{*}{ Afinn score } & -0.24 & 1.0 & -0.3 & -0.27 & -0.14 & -0.16 & -0.12 & 0.07 \\
\hline & -0.68 & 1.0 & -0.59 & -0.53 & -0.28 & -0.29 & -0.27 & 0.11 \\
\hline \multirow{2}{*}{ Political vector } & 0.4 & -0.3 & 1.0 & 0.6 & 0.36 & 0.38 & 0.22 & -0.12 \\
\hline & 0.58 & -0.59 & 1.0 & 0.7 & 0.45 & 0.48 & 0.27 & -0.21 \\
\hline \multirow{2}{*}{ Hate vector } & 0.45 & -0.27 & 0.6 & 1.0 & 0.21 & 0.23 & 0.22 & -0.0 \\
\hline & 0.67 & -0.53 & 0.7 & 1.0 & 0.28 & 0.31 & 0.29 & -0.01 \\
\hline \multirow{2}{*}{ Political interest } & 0.14 & -0.14 & 0.36 & 0.21 & 1.0 & 0.52 & 0.24 & -0.15 \\
\hline & 0.24 & -0.28 & 0.45 & 0.28 & 1.0 & 0.48 & 0.17 & -0.18 \\
\hline \multirow{2}{*}{ Political knowledge } & 0.17 & -0.16 & 0.38 & 0.23 & 0.52 & 1.0 & 0.23 & -0.27 \\
\hline & 0.22 & -0.29 & 0.48 & 0.31 & 0.48 & 1.0 & 0.17 & -0.3 \\
\hline \multirow{2}{*}{ Hostility } & 0.15 & -0.12 & 0.22 & 0.22 & 0.24 & 0.23 & 1.0 & -0.13 \\
\hline & 0.29 & -0.27 & 0.27 & 0.29 & 0.17 & 0.17 & 1.0 & -0.12 \\
\hline \multirow{2}{*}{ Female } & 0.0 & 0.07 & -0.12 & -0.0 & -0.15 & -0.27 & -0.13 & 1.0 \\
\hline & -0.05 & 0.11 & -0.21 & -0.01 & -0.18 & -0.3 & -0.12 & 1.0 \\
\hline
\end{tabular}

is Garg et al. (2018), who use word embeddings to study changes in gender and ethnic stereotypes over time.

It is important to note that we are not the first to demonstrate the usefulness of using word embeddings to study changes over time or across contexts. What we are claiming is that no currently existing method for measuring online hate specifically is able to do this. This is why the superunsupervised approach is an important contribution to the measurement of online political hostility. Although in principle expert raters in, for example, different countries could be used to train a classifier, it would be very costly and difficult to compare levels of political hate across countries. This is, in contrast, possible using word embeddings if the vector spaces are aligned; we elaborate on this in SM Section 1.1. It is, in principle, also possible to use newer methods utilizing context embeddings to study the changes in embeddings over time but in practice it is quite difficult since these methods require much more data than is available in most real life use cases; a recent study suggested that 10 million to 100 million words are needed (Zhang et al. 2020). We suggest the super-unsupervised represents a great alternative to these two techniques in many situations.

\subsection{Methods and measures}

To illustrate the usefulness of the the approach, we perform two analyses. First, we use our American Twitter data to create two classifiers for political hostility separately for participants identifying as either Republicans or Democrats. We then align their vector spaces, and investigate which words are differentially related to political hostility for these two subpopulations (see Section SM 1.5 for details).

Second, we use Twitter data collected before and after recent national elections in four countries (Denmark, Sweden, Germany, Italy) to demonstrate the approach's ability to study changes over 
time and across contexts. In each country, we follow our supervised-unsupervised approach but use local words for "political hate" to construct the word vectors (see SM Section 4 for details). ${ }^{8}$ We then examine over-time changes in political hate within each country. Intuitively, if our approach captures the relevant contextual features, we would expect to find that national levels of political hate raise as election days draw nearer before dropping as the political dust settles.

\subsection{Results}

Figure 3 reveals how strikingly differently Democrats and Republicans think about "political hate." Much in line with lay intuitions about modern-day cleavages in American politics, we find that Democrats are more likely than Republicans to associate political hate with "xenophobic", "islamophobic," and "nationalism", while Republicans, on the other hand, place emphasis on words like "left" and "leftist." It is also noteworthy that "(dis)loyalty," a moral foundation valued particularly by Republicans (Haidt 2013) show up. Furthermore "disgust" is also more associated with political hate for Republicans. This is in line with studies demonstrating greater disgust sensitivity for Republicans (Inbar, Pizarro, and Bloom 2009). These different conceptions of political hate across partisan subpopulations are important. Not only do they demonstrate just how differently people of different political stripes can understand the same political concepts; they also underscore one of our key theoretical arguments: Context matters significantly for how we understand words.

The second figure illustrates the development of political hate leading up to general elections in Sweden, Denmark, Germany and Italy. In all cases we can see that political hate reaches its maximum at the exact day of the election and that political hate is slowly increasing in the period leading up to the election. This validity check demonstrates our super-unsupervised approach is indeed picking up on political hate. But it also illustrates the power of the approach to study changes over time and across different contexts with little effort; an objective that would be difficult to accomplish with, say, dictionary-based approaches tailored to the English language.

8. The words used to measure "political" across Denmark, Sweden, Germany and Italy were "politik", "politik", "politik", and "politica" and the words used to measure "hate" were "had","hata","hassen" and "odiare". 


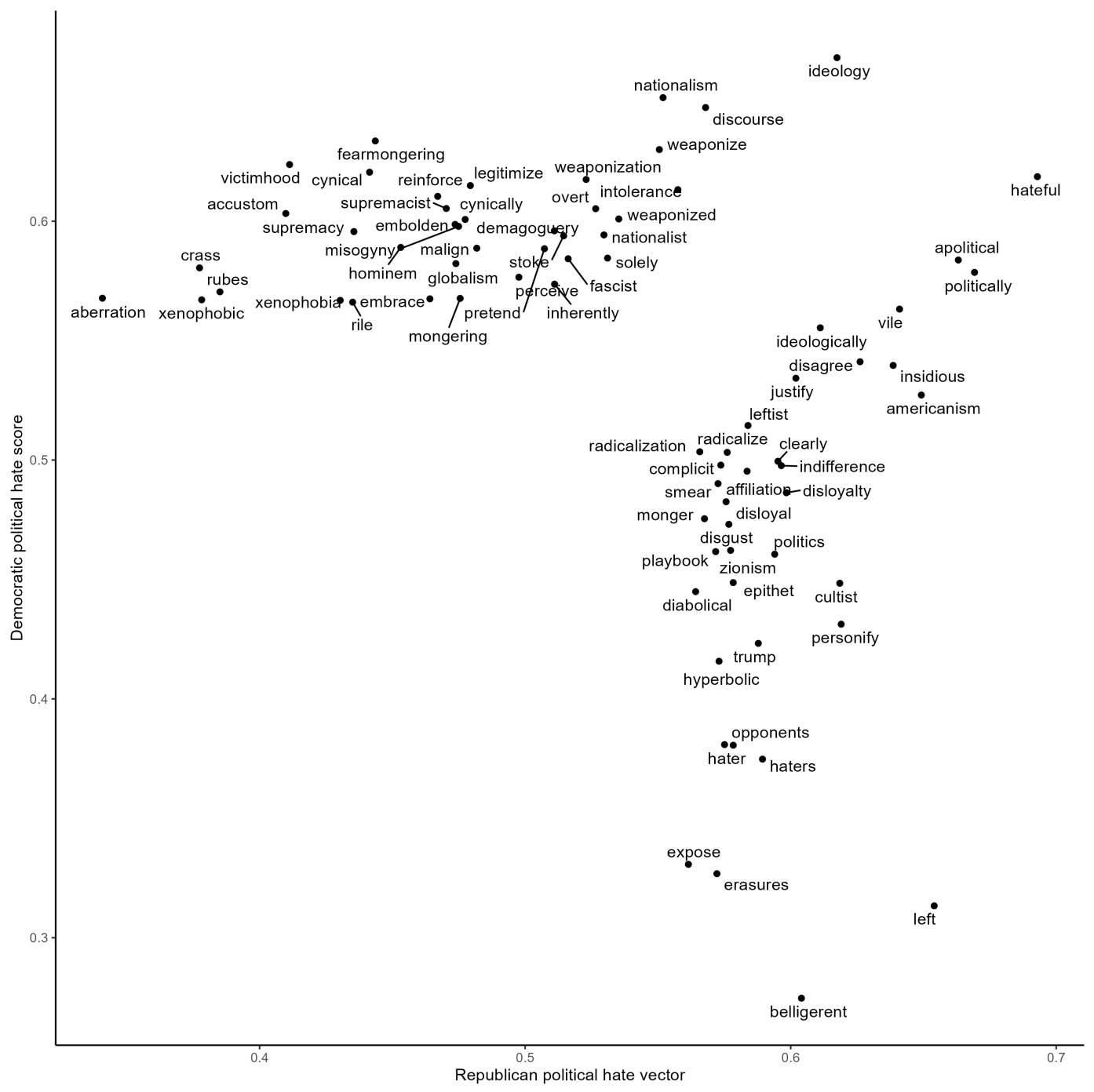

Figure 3. The figure illustrates the distance from each word to Political Hate for the Democratic and the Republican classifier of Political Hate. We have included the top 70 words for each group and excluded those where the difference in distance is smaller than .05 to highlight the main differences 

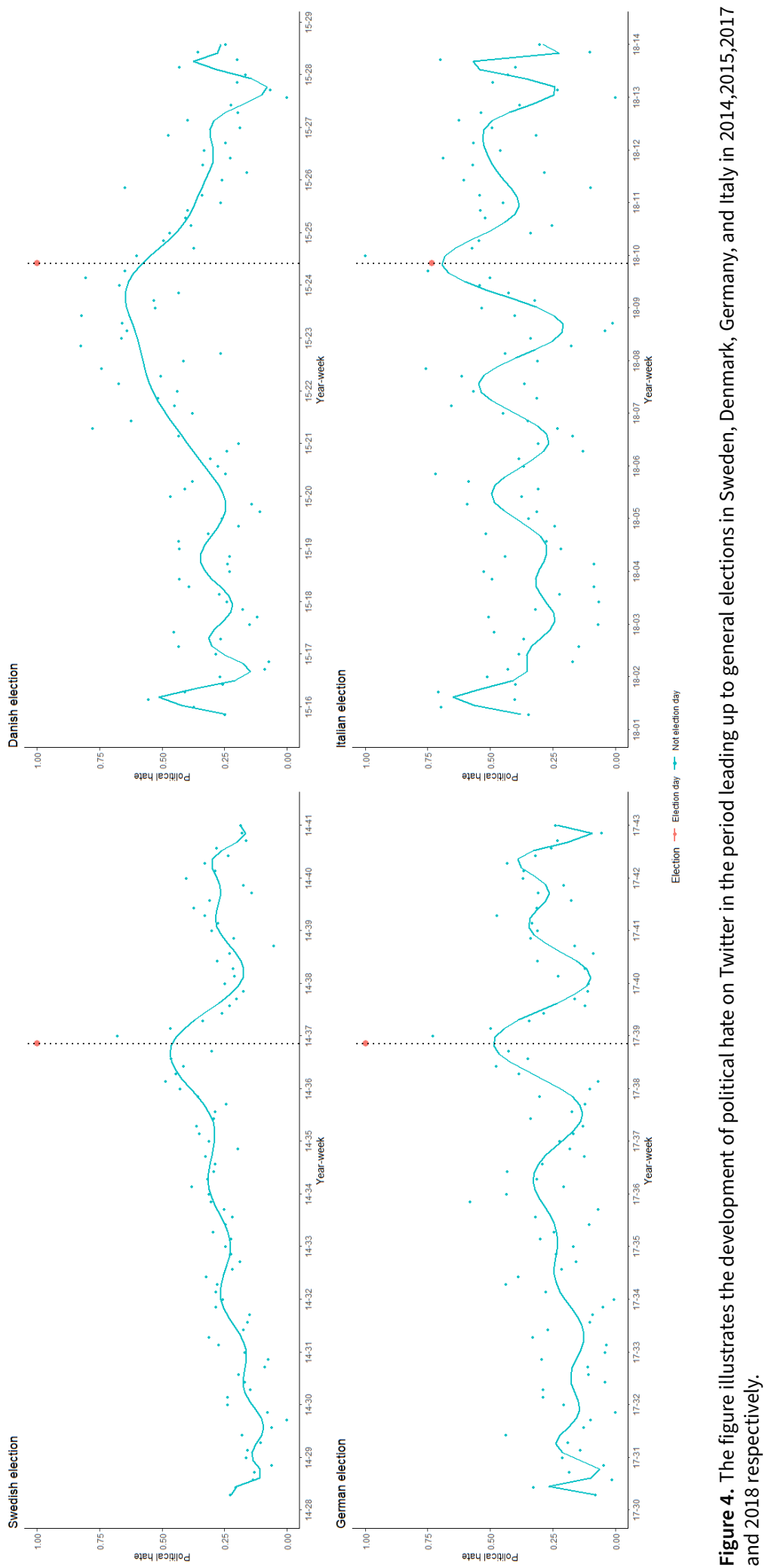


\section{Additional validity tests}

The full background, and some additional results, for the remaining validity tests, i.e. criterion validity, external validity and ecological validity can be found in SM Section 1.

\section{Conclusion and discussion}

Current approaches to studying online (political) hate are struggling with measurement and definition to such an extent that a recent review of the literature has called it (Siegel 2020, p 59): "clouded by definitional ambiguity". This has hindered a cumulative research program on the causes and consequences of online hate as well as efforts to fight its continued presence on social media platforms. In this manuscript, we have argued that the super-unsupervised approach to measuring and defining political hate has clear advantages compared to classic methods such as topic modeling approaches, dictionary-based approaches, and approaches using a classifier based on meticulous hand-labeling of texts. We first set up three standards for the measurement of online political hate and argued that they have clear advantages compared to current state-of-the-art approaches.

By using respondents' "self-labeling”, i.e. their everyday word usage in Twitter communications, we are able to attach the label "political hostility" to an otherwise completely unlabeled set of tweets. In this way, we can circumvent the high cost of hand-labeling, which haunts the supervised approach, and remedy the inability of the supervised approach to handle context-dependent labels. This also carries an important implication for current difficulties defining and measuring hate speech in online (social) media. If the users do not have a single clear idea of what hate speech is, trying to measure this as a single construct applicable to all contexts will always prove difficult, even when using very advanced NLP techniques. Researchers can of course try to define external "classifications" of hate speech using hand-labeling, but in a sense they are bound to fail if these conceptualizations do not to some extent correspond to the way users think and write about the construct - possibly in quite different ways in different situations.

The super-unsupervised approach not only provides meaningful "classifications" but also beats current standard methods used to measure online political hostility, such as dictionary-based approaches (afinn-scores) and model-based classifications (toxicity scores). To demonstrate this, we subjected the approach to five validity tests: face validity, convergent and discriminant validity and criterion validity, and in the appendix, we discuss the external validity and further ecological validity tests of the approach. In fact we are able to distinguish between the concepts of (1) "hate", (2) "politics" and (3) "political hate" using this approach, so depending on the researcher's need, there are several options to choose from. From a methodological point of view, this highlights the fact that the method can in fact be used to study related but distinct constructs with a fair amount of precision.

Furthermore, the approach has clear advantages in terms of the definition and conceptualization of political hate: We simply use the user's own perceptions of what constitutes a politically hateful text. By taking this approach, we side-step the almost insurmountable conceptual task of defining and conceptualizing what constitutes (political) hate online, which plagues dictionary-based approaches as well as approaches based on hand-labeling of texts.

Despite this, the approach and our validation face limitations. First, there is the practical limitation that the method requires a sample size of around 100,000 tweets to reliably estimate the effects found here. In some cases, it is possible to simply use pretrained embeddings, such as those available through pre-trained language models. This of course requires a suited language model for the problem at hand already exists, which may or may not pose a problem.

A second practical limitation of the present investigation is that we have primarily validated the approach using Twitter data. Twitter has over three hundred million users and is one of the world's most important online political platforms. It is relevant to assess this approach on other social media platforms such as Facebook; in completely different political contexts such as parliamentary debates; 
and even in non-political settings such as Reddit.

There are also relevant theoretical limitations relating to the super-unsupervised approach. This method utilizes the conception that words acquire meaning in the context in which they appear and is thus an improvement over simple word counts such as dictionary-based methods (e.g. afinn scores). However, the word2vec method still does not take word order into account (Chang and Masterson 2020) and more advanced grammatical relationships that might affect the meaning of words (Hewitt and Manning 2019; Clark et al. 2019). As discussed above more advanced pre-trained language models, such as Google's BERT exist, which did not provide better or worse results than the super-unsupervised approach in terms of convergent and discriminant validity. When data is plentiful, i.e. in the hundreds of millions, we would however recommend training these models from scratch to achieve a more nuanced and fine-grained understanding of political hate.

Given these limitations and opportunities, what can the method be used for? It definitely seems useful for measuring and classifying tweets into "hate," "political" and "political hate." It can also be used as a first step in hand-labeling. Imagine a study of the frequency of hate speech using a strict legal definition. The use of respondents' self-labeling would not be sufficient, but it could serve as a powerful first screening step, which could provide expert coders with a sample to code. Instead of having to go through millions and millions of tweets, this could serve as a first rough attempt at separating tweets into hateful and non-hateful. This could be combined with, e.g., the active-labeling approach of Miller, Linder, and Mebane (2020).

In general, we suggest that it can be used to measure any construct that is also used in natural language and is distinct enough. This is especially true when the data are plentiful, i.e., in the millions, such as is often the case with online social media data. Here, the abundance of data is both an advantage for the super-unsupervised approach and an obstacle to supervised approaches as the sheer size will limit the possibilities of hand-labeling. Future studies should thus seek to validate the approach for other constructs in addition to "political hostility".

\section{Data Availability Statement}

All scripts, and data that is not personally identifiable, will be made available on OSF upon publication.

\section{References}

Barberá, Pablo, Amber E. Boydstun, Suzanna Linn, Ryan McMahon, and Jonathan Nagler. 2021. Automated text classification of news articles: a practical guide. Political Analysis, 1-24.

Blei, David M, Andrew Y Ng, and Michael I Jordan. 2003. Latent dirichlet allocation. Journal of machine Learning research 3 (Jan): 993-1022.

Brandt, Mark J, Christine Reyna, John R Chambers, Jarret T Crawford, and Geoffrey Wetherell. 2014. The ideologicalconflict hypothesis: intolerance among both liberals and conservatives. Current Directions in Psychological Science 23 (1): 27-34.

Campbell, Donald T, and Donald W Fiske. 1959. Convergent and discriminant validation by the multitrait-multimethod matrix. Psychological bulletin 56 (2): 81.

Chang, Charles, and Michael Masterson. 2020. Using word order in political text classification with long short-term memory models. Political Analysis 28, no. 3 (July): 395-411.

Clark, Kevin, Urvashi Khandelwal, Omer Levy, and Christopher D Manning. 2019. What does bert look at? an analysis of bert's attention. arXiv preprint arXiv:1906.04341.

Costello, Matthew, and James Hawdon. 2018. Who are the online extremists among us? sociodemographic characteristics, social networking, and online experiences of those who produce online hate materials. Violence and gender 5 (1): 55-60.

Cranmer, Skyler J., and Bruce A. Desmarais. 2017. What can we learn from predictive modeling? Political Analysis 25 (2): 145-166. https://doi.org/10.1017/pan.2017.3.

Cronbach, Lee J, and Paul E Meehl. 1955. Construct validity in psychological tests. Psychological bulletin 52 (4): 281. 
Davidson, Thomas, Dana Warmsley, Michael Macy, and Ingmar Weber. 2017. Automated hate speech detection and the problem of offensive language. In Proceedings of the 11th international aaai conference on web and social media, 512-515. ICWSM '17. Montreal, Canada.

De Saussure, Ferdinand. 2011[1916]. Course in general linguistics. Columbia University Press.

DeFranza, David, Himanshu Mishra, and Arul Mishra. 2020. How language shapes prejudice against women: an examination across 45 world languages. Journal of personality and social psychology.

Devlin, Jacob, Ming-Wei Chang, Kenton Lee, and Kristina Toutanova. 2018. Bert: pre-training of deep bidirectional transformers for language understanding. arXiv preprint arXiv:1810.04805.

Djuric, Nemanja, Jing Zhou, Robin Morris, Mihajlo Grbovic, Vladan Radosavljevic, and Narayan Bhamidipati. 2015. Hate speech detection with comment embeddings. In Proceedings of the 24th international conference on world wide web, 29-30.

Duarte, José L, Jarret T Crawford, Charlotta Stern, Jonathan Haidt, Lee Jussim, and Philip E Tetlock. 2015. Political diversity will improve social psychological science 1. Behavioral and Brain Sciences 38.

Firth, J. 1957. Studies in linguistic analysis. Oxford.

Garg, Nikhil, Londa Schiebinger, Dan Jurafsky, and James Zou. 2018. Word embeddings quantify 100 years of gender and ethnic stereotypes. Proceedings of the National Academy of Sciences 115 (16): E3635-E3644.

Griezel, Lucy, Linda R Finger, Gawaian H Bodkin-Andrews, Rhonda G Craven, and Alexander Seeshing Yeung. 2012. Uncovering the structure of and gender and developmental differences in cyber bullying. The Journal of Educational Research 105 (6): 442-455.

Grimmer, Justin. 2010. A bayesian hierarchical topic model for political texts: measuring expressed agendas in senate press releases. Political Analysis 18 (1): 1-35.

Haidt, Jonathan. 2013. The righteous mind: why good people are divided by politics and religion. Vintage.

Hewitt, John, and Christopher D Manning. 2019. A structural probe for finding syntax in word representations. In Proceedings of the 2019 conference of the north american chapter of the association for computational linguistics: human language technologies, volume 1 (long and short papers), 4129-4138.

Inbar, Yoel, David A Pizarro, and Paul Bloom. 2009. Conservatives are more easily disgusted than liberals. Cognition and emotion 23 (4): 714-725.

JIGSAW. https://perspectiveapi.com/\#/home.

Jost, John T, Christopher M Federico, and Jaime L Napier. 2009. Political ideology: its structure, functions, and elective affinities. Annual review of psychology 60:307-337.

Kim, Jin Woo, Andrew Guess, Brendan Nyhan, and Jason Reifler. 2021. The distorting prism of social media: how selfselection and exposure to incivility fuel online comment toxicity. Journal of Communication 71 (6): 922-946.

Matthews, Gerald, Ian J Deary, and Martha C Whiteman. 2003. Personality traits. Cambridge University Press.

Meredith, William. 1993. Measurement invariance, factor analysis and factorial invariance. Psychometrika 58 (4): 525-543.

Miller, Blake, Fridolin Linder, and Walter R Mebane. 2020. Active learning approaches for labeling text: review and assessment of the performance of active learning approaches. Political Analysis 28 (4): 532-551.

Nielsen, Finn Årup. 2011. A new anew: evaluation of a word list for sentiment analysis in microblogs. arXiv preprint arXiv:1103.2903.

Rheault, Ludovic, and Christopher Cochrane. 2020. Word embeddings for the analysis of ideological placement in parliamentary corpora. Political Analysis 28 (1): 112-133.

Roberts, Margaret E, Brandon M Stewart, Dustin Tingley, Edoardo M Airoldi, et al. 2013. The structural topic model and applied social science. In Advances in neural information processing systems workshop on topic models: computation, application, and evaluation, vol. 4. Harrahs and Harveys, Lake Tahoe.

Rodman, Emma. 2020. A timely intervention: tracking the changing meanings of political concepts with word vectors. Political Analysis 28 (1): 87-111.

Rodriguez, Axel, Carlos Argueta, and Yi-Ling Chen. 2019. Automatic detection of hate speech on facebook using sentiment and emotion analysis. In 2019 international conference on artificial intelligence in information and communication (icaiic), 169174. IEEE.

Schmuckler, Mark A. 2001. What is ecological validity? a dimensional analysis. Infancy 2 (4): 419-436. 
Siegel, Alexandra A, Evgenii Nikitin, Pablo Barberá, Joanna Sterling, Bethany Pullen, Richard Bonneau, Jonathan Nagler, Joshua A Tucker, et al. 2021. Trumping hate on twitter? online hate speech in the 2016 us election campaign and its aftermath. Quarterly Journal of Political Science 16 (1): 71-104.

Siegel, Alexandra A. 2020. Online hate speech. In Social media and democracy: the state of the field, prospects for reform, edited by Nathaniel Persily and Joshua A Tucker. Cambridge University Press.

Stine, Robert A. 2019. Sentiment analysis. Annual review of statistics and its application 6:287-308.

Törnberg, Anton, and Petter Törnberg. 2016. Muslims in social media discourse: combining topic modeling and critical discourse analysis. Discourse, Context \& Media 13:132-142.

Waldron, Jeremy. 2012. The harm in hate speech. Harvard University Press.

Wich, Maximilian, Jan Bauer, and Georg Groh. 2020. Impact of politically biased data on hate speech classification. In Proceedings of the fourth workshop on online abuse and harms, 54-64. Online: Association for Computational Linguistics, November. https://doi.org/10.18653/v1/2020.alw-1.7. https://aclanthology.org/2020.alw-1.7.

Zhang, Yian, Alex Warstadt, Haau-Sing Li, and Samuel R Bowman. 2020. When do you need billions of words of pretraining data? arXiv preprint arXiv:2011.04946. 